\title{
The Joint Extraction Algorithm Based on Color and Depth Information
}

\author{
Zijuan Zhang \\ Shaanxi Polytechnic Institute, Xianyang, 712000, China \\ Email address: bluesky1023@126.com
}

\section{Keywords: Depth Map, Importance Map, Extraction Algorithm, Image Retargeting}

Abstract. For importance map, most image retargeting algorithms use color information only (such as the gradient map or saliency map, etc.) to deal with this problem. This paper considers depth information based on the analysis of the depth map besides, combines with color information and puts forward a combined color depth information's importance map. The importance map can accurately identify the subject of source information. Guided by this importance map, retargeting results can protect the important areas in the source image better and obtain the better effects.

\section{Introduction}

Image retargeting algorithm can be divided into two stages: the perception stage is the extraction of the importance map for the source information. The recognition accuracy of the importance map directly affects the resizing results. For important map, most retargeting algorithm use color information only (such as the gradient map or saliency map, etc.) to deal with the problem. The important map based on color information reflects the human eye's focus area not quite accurately, its precision still need to be improved. In view of this, based on physiology[1] and psychology theory [2-4], this paper considers depth information increasingly, compared with other automatic method to calculate the important map, qualitative analysis and quantitative results show that this algorithm can extract the important map with higher accuracy.

\section{Joint Extraction Algorithm}

The combination of depth information and color information, its extraction algorithm's process is shown in figure 1 . The source image comes from the RGB-D device. Algorithm was divided into four steps: edge detection, saliency test, depth map generation and the extraction of important map. Among them, the edge detection and saliency test belong to the category of color information processing, depth map generation falls within the scope of the depth information processing, important map's extraction not only considers the color information in the source information (gradient map and saliency map), but also combines the depth information (depth map).

\section{The edge detection and saliency test}

For edge and saliency detection, this paper uses sobel operator[5] to detect the gradient map, adopts Itti algorithm[6] to detect significant map. Algorithm firstly converts RGB color images to grayscale image, and then conducts on gray image for edge detection and saliency test. Edge detection is realized by using the Sobel operator, makes the extracted features to ensure the continuity on the edge of the source image. Sobel operator is discrete difference operator and was used to calculate the image brightness function's approximation. In any pixel of the image by using this operator, we will produce a corresponding gradient vector. The operator contains two sets of matrices respectively: the horizontal and vertical operator, which was used with image's plane convolution, we can calculate the brightness of the horizontal and vertical difference approximation.

Supposing the original image is $I, M_{x}$ and $M_{y}$ are the horizontal and vertical edge detection of image respectively, then 


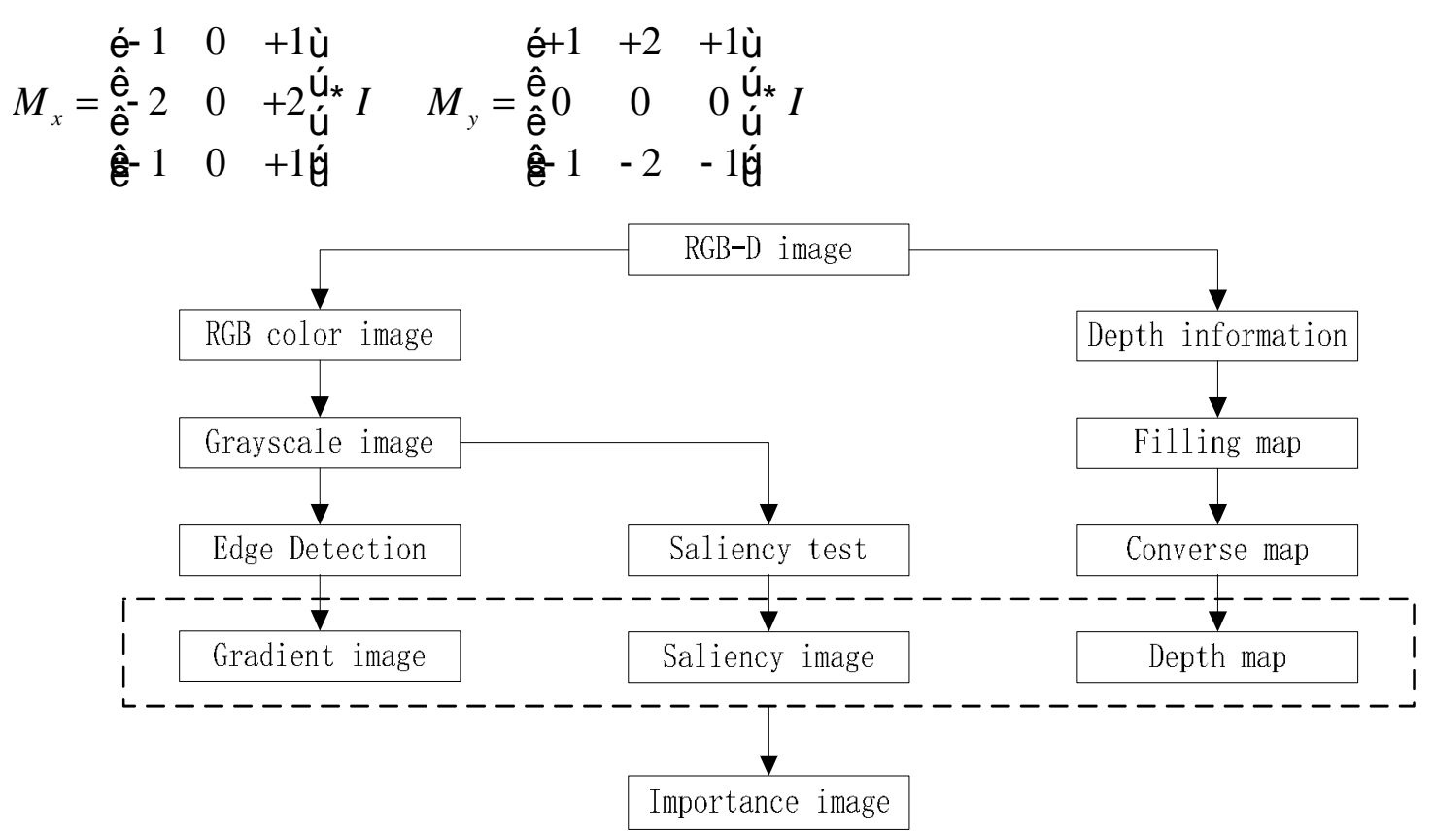

Figure 1 The importance map of the combination of color and depth information

Among them, * on behalf of the convolution; The transverse and longitudinal gradient approximation of each pixel in the image is calculated by type (1), the gradient $M_{\text {gradient }}$ is

$$
M_{\text {gradient }}=\sqrt{M_{x}^{2}+M_{y}^{2}}
$$

The gradient direction $\theta$ is

$$
\theta=\arctan \left(\frac{M_{y}}{M_{x}}\right)
$$

If $\theta=0$, it suggests that there has vertical edge image. Figure 2 (b) is obtained by using the Sobel operator in the original image (figure 2) (a) for getting the gradient map, which reflects the original image's edge character.

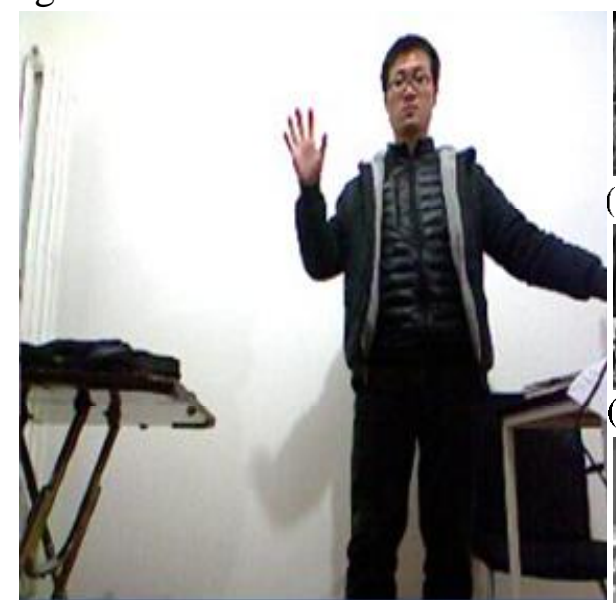

(a) Original image

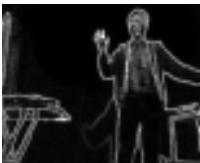

(b) Gradient map

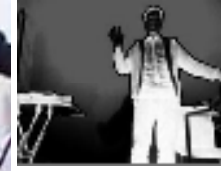

(c) Saliency map

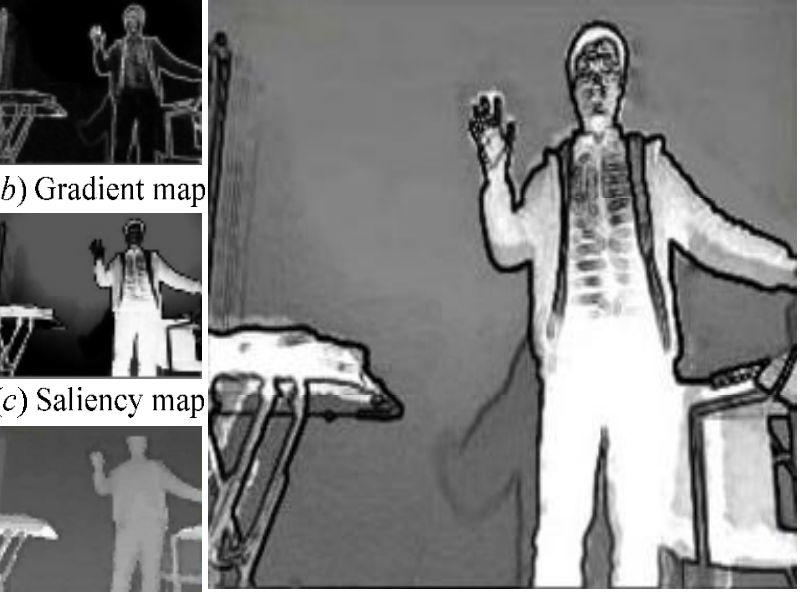

(d) Depth map

(e) Importance map

Figure 2 The example of the extraction of importance $\operatorname{map}(c=1, \gamma=0.4)$

For saliency detection, it is used Itti algorithm [6] here. Itti model is based on human visual characteristics, combines with the knowledge of psychology and biology, uses the reaction of human eye to color, brightness, and the direction which belong to the underlying information, simulates the human eye test of significance from the bottom to up. Figure 2 (c) is saliency map which is based on the Itti model for checking the original image. Saliency map reflects the basic human eyes area, but the grey value of face, collar information is less than the gray value of the wall. Therefore the information reflected by the saliency map is not accurate, it remains to be further optimized. 


\section{The extraction of importance map}

Importance map is used to guide the image retargeting. In order to extract the importance map of source images, this algorithm combined the gradient map, saliency map and depth map to extract important map $M_{\text {depthbased }}$

$$
M_{\text {depthbased }}=\left(M_{\text {gradient }}+M_{\text {saliency }}\right) D_{\text {meanshift }}
$$

$M_{\text {gradient }}$ and $M_{\text {saliency }}$ is the gradient map and saliency map respectively, $D_{\text {meanshift }}$ is the depth map. The main purpose of the joint method is that inhibit pixels of far away from the camera achieve high importance.

After extracting the importance map $M_{\text {depthbased }}$, we use this method:

$$
s=c r^{\gamma}
$$

Among (5), $r$ is the importance map, $s$ is the importance map by adjusting the original importance map. $c$ and $\gamma$ is the positive parameter. When $\gamma$ is increased, the main subject information is decreased. On contrast, when $\gamma$ is decreased, the main subject information is increased. Since the goal of this algorithm is protection of main body information, we can set $\gamma<1$. Figure 2 shows the method in this paper which combined the gradient map, saliency and depth map (figure 2 (d)) for extracting the source image's importance map(figure 2 (e)) $(c=1, \gamma=0.4)$.

\section{Summary}

This paper proposes a joint extraction algorithm of color and depth information for important map, an important map was obtained by the joint of gradient map, saliency map and depth map. The map which was extracted by new algorithm has higher accuracy. Retargeting result not only reduces the deformation of the subject, but also allows greater distortion in an important area.

\section{References}

[1] Fink G.R., Dolan R.J., Halligan P.W., et al. Space-based and object-based visual attention: shared and specific neural domains, J. BRAIN. 120 (1997) 2013-2028

[2] Eriksen B.A., Eriksen C.W. Effects of noise letters upon the identification of a target letter in a nonsearch task, J. Perception and Psychophysics. 16 (1974) 143-149

[3] Eriksen C.W., Hoffman J.E. The extent of processing of noise elements during selective encoding from visual displays, J. Perception and Psychophysics. 14 (1973) 155-160

[4] Fox E. Perceptual grouping and visual selective attention, J. Perception and Psychophysics. 60 (1998) 1004-1021

[5] Rafael C.G., Richard E.W. Digital image processing, Second Edition. Publishing House of Electronics Industry, BEIJING, 2010.

[5] Itti L., Koch C., Ernst E. A model of saliency-based visual attention for rapid scene analysis, J. IEEE Transactions on Pattern Analysis and Machine Intelligence. 20(1998): 1254-1259 